\title{
Hallazgo de Hirundichthys rondeletii (Valenciennes, 1847) en Ancud, Chiloé, con datos sobre peces voladores en colecciones de Chile (Osteichthyes: Exocoetidae)
}

\author{
Rodrigo Vera S. y Germán Pequeño R. \\ Instituto de Zoología "Ernst F. Kilian”, Universidad Austral de Chile \\ Casilla 567, Valdivia, Chile \\ E-mail: rfvera@ hotmail.com; gpequeno@uach.cl
}

Recibido: 27 diciembre 2001; versión corregida: 1 agosto 2002; aceptado: 23 septiembre 2002

\begin{abstract}
RESUMEN. Se comunica el hallazgo de un ejemplar del pez volador, Hirundichthys rondeletii, capturado accidentalmente frente a Ancud $\left(c a .42^{\circ} \mathrm{S}, 74^{\circ} \mathrm{W}\right)$, Chile, que constituye el registro más austral de esta especie en el Pacífico suroriental. Este ejemplar se compara morfológicamente con ejemplares de otras colecciones. Se discuten las posibles causas de su aparición en esa latitud austral y se entregan datos de catalogación y origen geográfico de otros ejemplares de la familia Exocoetidae, depositados en colecciones chilenas.
\end{abstract}

Palabras claves: pez volador, Exocoetidae, Hirundichthys, Ictiogeografía, Chile.

\section{Finding of Hirundichthys rondeletii (Valenciennes, 1847) in Ancud, Chiloé, with data on flying fishes in collections of Chile (Osteichthyes: Exocoetidae)}

\begin{abstract}
The finding of one specimen of the flying fish Hirundichthys rondeletii, accidentally captured off Ancud $\left(42^{\circ} \mathrm{S}, 74^{\circ} \mathrm{W}\right.$ aprox $)$, Chile, is communicated. This is the southernmost record for the species in the southeastern Pacific. The specimen is analyzed and morphologically compared with other conspecific from collections. The possible causes of its appearance in such southern latitude are discussed and catalog data including location of other specimens of the family Exocoetidae preserved in collections of Chile are given.
\end{abstract}

Key words: flying fish, Exocoetidae, Hirundichthys, ichthyogeography, Chile.

Los peces voladores viven en aguas superficiales del mar abierto, así como en zonas costeras. Son ampliamente conocidos por su capacidad de saltar fuera del agua y planear por el aire distancias considerables, con ayuda de sus aletas pectorales expandidas (planeadores de dos alas) y a veces también con sus aletas pélvicas (planeadores de cuatro alas). Aunque estos peces son en general apreciados como alimento humano, en la actualidad existen pocas pesquerías que los explotan. La mayoría de las especies son atraídas por la luz. Los peces de la familia Exocoetidae se caracterizan por ser alargados, de cuerpo cilíndrico, hocico grueso generalmente romo y más corto que el ojo (excepto en Fodiator). Mandíbulas con dientes muy pequeños o sin dientes; branquispinas bien desarrolladas. Aletas sin espinas, la dorsal y la anal en posición muy posterior, sus bases cortas y opuestas; aletas pectorales extraordinariamente largas y sobrepasan el origen de la aleta dorsal; aletas pélvicas abdominales muy grandes en algunas especies; aleta caudal ahorquillada y su lóbulo inferior es más largo que el superior. Línea lateral de posición muy baja en el cuerpo; escamas grandes, cicloideas y deciduas. Dorso oscuro, vientre claro, en peces vivos los colores oscuros son generalmente azul o verde iridiscentes. Aletas 
pectorales en algunas especies con manchas oscuras o franjas pálidas. Existen barbillas mentonianas en los juveniles de muchas especies (Parin en Fischer et al., 1995).

En el Pacífico suroriental y especialmente en Chile, las referencias sobre la familia Exocoetidae en su conjunto son muy escasas. Fowler (1942) resume la sinonimia en su época; Mann (1954) la incluye en una obra general, pero sin mayor análisis; De Buen (1961) presenta una revisión y Pequeño (1989) considera 12 especies, entre ellas Hirundichthys rondeletii. Meléndez et al. (1993), entregan los datos de catalogación de las especies representadas en el Museo Nacional de Historia Natural de Chile. Así, los peces de la familia Exocoetidae que habitan aguas chilenas no se han estudiado en su conjunto. Esta puede ser una razón que explica su desconocimiento científico en el Pacífico suroriental. De allí que se considere necesario comunicar cualquier hallazgo que aporte nuevos datos sobre las especies y su distribución geográfica. El presente trabajo informa el hallazgo de un ejemplar de pez volador capturado en el litoral de Chiloé. Además, entrega información sobre los peces voladores preservados en colecciones chilenas, incluyendo datos básicos de recolección.

El hallazgo del pez se produjo frente Faro Corona, Ancud, isla Chiloé, Chile ( $\left.c a .42^{\circ} \mathrm{S}, 74^{\circ} \mathrm{W}\right)$. En el momento de su captura se fijó en formalina y al ingresar a la colección de la Universidad Austral de Chile, se preservó en alcohol al $70 \%$ en septiembre de 2001; IZUA-PM- 2245. Este pez cayó dentro del bote de pesca, sorpresivamente, mientras "volaba" y por eso, su captura se considera accidental.

Se estudió su morfometría, principalmente relacionada con su longitud estándar (LE). Además, con un pie de metro con $\pm 0,1 \mathrm{~mm}$ de precisión, se efectuaron las siguientes mediciones: longitud total (LT), longitud furcal (LF), longitud de la cabeza (LC), diámetro orbital (DO), distancia interorbital (DI), altura máxima del cuerpo (AM), ancho máximo del cuerpo (AMC), longitud predorsal (LPr), longitud preanal (LPA), altura del pedúnculo caudal (APD), longitud de la aleta pectoral (LP), longitud de la aleta pélvica (LPL), longitud de la base de la aleta dorsal (LBD), longitud de la base de la aleta anal (LBA), distancia preorbital (DP), distancia postorbital (DPO) y longitud de la mandíbula superior (LMS). También se calcularon las siguientes relaciones: LE/ LC; LE/AM; LE/LP y LP/LC.

Además de estas mediciones se hizo el recuento del número de radios de las aletas dorsal, anal, pectoral, pélvica y caudal. La cuenta del número de escamas predorsales, que en el caso de este género es importante en el reconocimiento de las especies, no se realizó debido a que el ejemplar presentaba descamación en esa zona. La cuenta del número de vértebras, que también es un carácter que puede determinar la especie, no se efectuó por disponer sólo de un ejemplar y los intentos de radiografías resultaron deficientes.

La identificación del ejemplar (Fig. 1) se realizó con el apoyo de los trabajos de Chirichigno (1998), Heemstra y Parin (1986) y Fischer et al. (1995). Para los acrónimos institucionales, se siguió a Leviton et al. (1985), con excepción de la colección de la Universidad de Antofagasta. También para fines comparativos, se contó con un ejemplar de Hirundichthys rondeletii IZUA-PM-1448 capturado el 7 de octubre de 1991 alrededor de los 2855' S, 79³2'W.

El ejemplar analizado tiene las aletas pectorales muy largas, llegando más allá del extremo posterior de la base de la aleta dorsal; línea lateral sin rama pectoral; aletas pélvicas largas y sus extremos sobrepasan el origen de la anal; su punto de inserción es más cercano al origen de la anal que al punto de inserción de las pectorales. Origen de la aleta anal levemente por delante del origen de la dorsal. Dorsal con un número menor de radios que la anal (un radio). Primeros dos radios pectorales no ramificados y la membrana que los une sin curva pronunciada; palatinos sin dientes. LC 3,9 veces en la LP; LP 1,2 veces en la LE.

Por necesidades del reconocimiento se obtuvieron las siguientes relaciones: LE/LC: 4,8; LE/AM: 5,6; LE/LP: 1,2; LE/LC: 3,9. Las mediciones y recuentos efectuados, comparadas con otro espécimen de las colecciones, aparecen en la Tabla 1. Con todos estos antecedentes y según claves de reconocimiento, se concluyó que es un ejemplar de Hirundichthys rondeletii. La revisión de colecciones de peces chilenos y de los registros geográficos de la literatura, indican que este ejemplar, junto con el utilizado para la comparación, son los únicos que se preservan y documentan la presencia de $H$. rondeletti en Chile. El ejemplar objeto de esta nota, constituye el registro más austral para la especie.

La temperatura superficial del mar promedio histórico de abril para Puerto Montt (412' S, $72^{\circ} 57^{\prime} \mathrm{W}$ ), una localidad cercana a Ancud de la cual se tienen registros por años, es de $12,6^{\circ} \mathrm{C}$. La mínima para abril de 1991 ocurrió el día 18 con $11,1^{\circ} \mathrm{C}$ y 


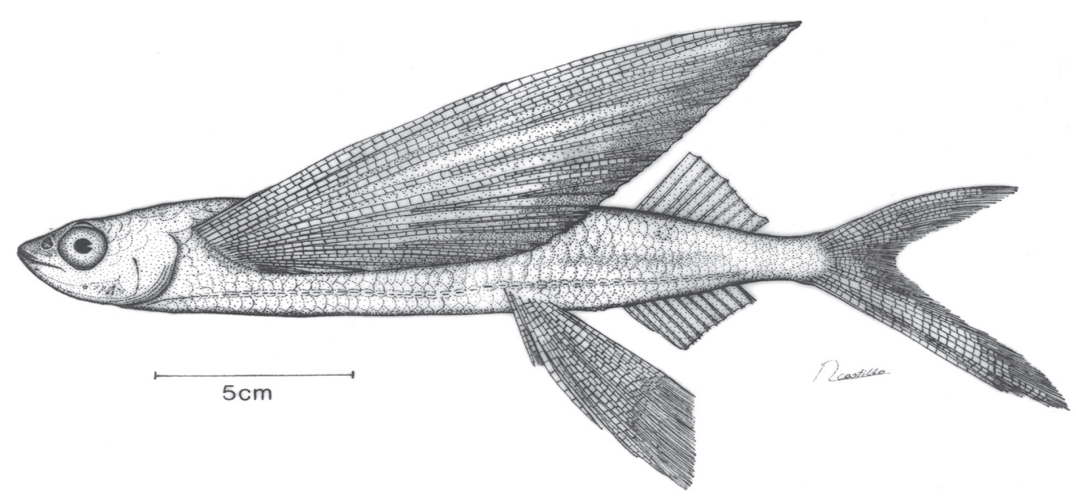

Figura 1. Hirundichthys rondeletii, IZUA-PM-2245, 268 mm LT.

Figure 1. Hirundichthys rondeletii, IZUA-PM-2245, 268 mm TL.

Tabla 1. Medidas (cm) y recuentos de Hirundichthys rondeletii de Ancud (IZUA-PM-2245) y otro especimen conespecífico para comparación (IZUA-PM-1448).

Table 1. Measurements (cm) and counts made on Hirundichthys rondeletii from Ancud (IZUA-PM-2245) and other conspecific comparative specimen (IZUA-PM-1448).

\begin{tabular}{|c|c|c|}
\hline Características & IZUA-PM- 2245 & IZUA-PM- 1448 \\
\hline \multicolumn{3}{|l|}{ Morfométricas } \\
\hline Longitud total (LT) & 26,8 & 31,3 \\
\hline Longitud furcal (LF) & 21,7 & 26,1 \\
\hline Longitud estándar (LE) & 20,4 & 24,0 \\
\hline Longitud de la cabeza (LC) & 4,2 & 4,7 \\
\hline Diámetro orbital (DO) & 1,4 & 1,4 \\
\hline Diámetro interorbital (DI) & 1,8 & 1,9 \\
\hline Altura máxima del cuerpo (AM) & 3,6 & 3,4 \\
\hline Ancho máximo del cuerpo (AMC) & 2,7 & 2,4 \\
\hline Distancia predorsal (DPD) & 15,3 & 16,9 \\
\hline Distancia preanal (DPA) & 14,9 & 17,2 \\
\hline Altura máxima pedúnculo caudal (AMPC) & 1,2 & 1,3 \\
\hline Longitud de la aleta pectoral (LP) & 16,4 & 18,7 \\
\hline Longitud aleta pélvica (LPL) & 7,7 & 7,9 \\
\hline Longitud de la base de la aleta dorsal (LBD) & 3,2 & 4,2 \\
\hline Longitud de la base de la aleta anal (LBA) & 3,2 & 4,1 \\
\hline Distancia preorbital (DP) & 0,9 & 1,0 \\
\hline Distancia postorbital (DPO) & 2,1 & 2,1 \\
\hline Longitud de la mandíbula superior (LMS) & 1,4 & 1,4 \\
\hline \multicolumn{3}{|l|}{ Merísticas (radios de aletas) } \\
\hline Dorsal & 10,0 & 12,0 \\
\hline Anal & 11,0 & 12,0 \\
\hline Pectoral & 17,0 & 17,0 \\
\hline Pélvica & 7,0 & 6,0 \\
\hline Caudal & 22,0 & 22,0 \\
\hline
\end{tabular}


la máxima fue el día 24 con $14,6^{\circ} \mathrm{C}$.

La morfología externa y recuento de las estructuras del espécimen estudiado, así como aquel utilizado para la comparación, coinciden con las descripciones y claves que hay en la literatura para reconocer a $H$. rondeletii (Bruun, 1935; Heemstra y Parin, 1986; Fischer et al., 1995; Chirichigno, 1998). Sin embargo, existen diferencias que es oportuno mencionar. En los dibujos de $H$. rondeletii que aparecen en los trabajos de Fischer et al. (1995), Chirichigno (1998) y Heemstra y Parin (1986), se aprecian diferencias evidentes entre uno y otro, en cuanto a forma de la aleta pectoral y su longitud, al igual que en la aleta pélvica. La proporción entre la altura y la longitud total también es diferente en cada uno de los dibujos. Esto difiere de la descripción dada en cada uno de los textos donde los tres autores coinciden en los criterios de identificación de la especie.

Según la figura de Heemstra y Parin (1986), la concavidad de la membrana entre los dos primeros radios (no ramificados) de la aleta pectoral es notoriamente pronunciada, lo que no ocurre en el ejemplar de $H$. rondeletii estudiado en este caso (Fig. 1). Cabe notar que Fischer et al. (1995) ilustraron su trabajo con la figura de Bruun (1935). La implantación de la aleta pectoral, en el dibujo de Chirichigno (1998) se encuentra detrás del opérculo, a diferencia del dibujo de Heemstra y Parin (1986). La longitud de la aleta pectoral de nuestro $H$. rondeletii difiere de los dibujos de Chirichigno (1998) y de Heemstra y Parin (1986), y se asemeja más al de Bruun (1935) y Fischer et al. (1995). La descamación observada en la parte dorsal del espécimen, es común en este tipo de peces con escamas deciduas (Fischer et al., 1995) (Fig. 2).

Aparentemente, la primera men-

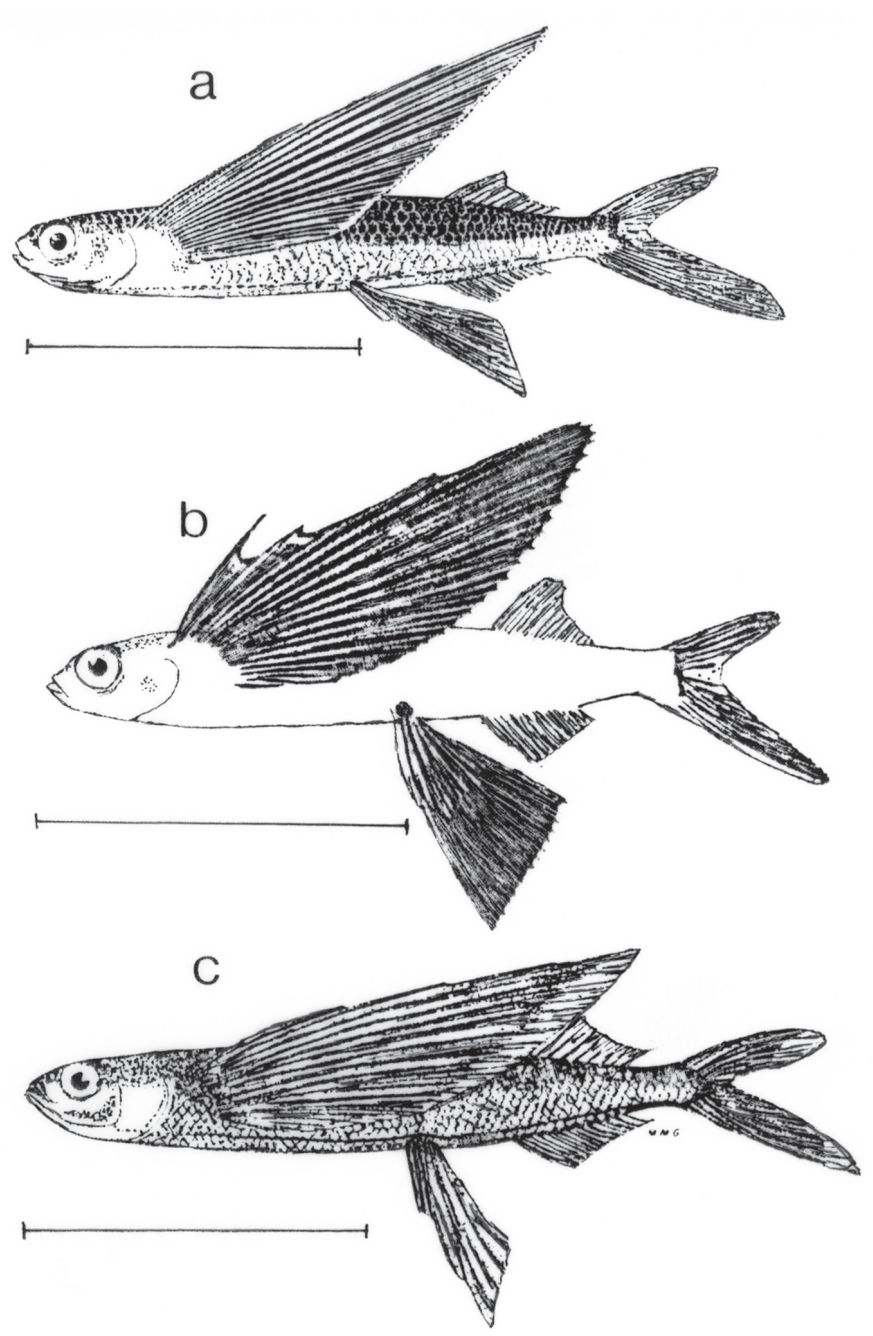

Figura 2. Dibujos de Hirundichthys rondeletii, ligeramente modificados de: a) Bruun (1935), b) Heemstra y Parin (1986) y c) Chirichigno (1998). Las escalas en a y c indican $10 \mathrm{~cm}$; en b indican $5 \mathrm{~cm}$.

Figure 2. Representative drawings of Hirundichthys rondeletii, slightly modified from: a) Bruun (1935), b) Heemstra and Parin (1986) and c) Chirichigno (1998). Scales in a and c indicate $10 \mathrm{~cm}$; in b indicate $5 \mathrm{~cm}$.

ción de H. rondeletti para Chile corresponde a Bahamonde y Pequeño (1975), quienes incluyen esta especie en una lista sistemática, sin mayores comentarios, pero basados en un trabajo de Parin (1969), quien menciona a la especie para el epipelágico de una vasta región estudiada aproximadamente, entre Panamá y Valparaíso. Parin (1969) indica que: "el tropical Hirundichthys rondeletti, el cual se encuentra en todos los océanos a lo largo de los márgenes de la región tropical, fue el más común en el área investigada. Este pez fue continuamente encontrado en $25^{\circ} \mathrm{S}$ a temperaturas de $17-20^{\circ} \mathrm{C}$, pero estuvo ausente de áreas costeras enfriadas como 
resultado de la surgencia de aguas profundas hacia la superficie". También, Parin (1960a) señala que $H$. rondeletii ha sido encontrado cerca de las costas de Japón, China y California en el hemisferio norte y a lo largo de Perú y Chile en el hemisferio sur. Los especímenes del hemisferio norte se capturaron en otoño alrededor de $\operatorname{los} 30-40^{\circ} \mathrm{N}, 143-165^{\circ} \mathrm{E}$, y probablemente en invierno, esta especie migra hacia el sur para invernar y desovar.

Parin (1960b) agrega que $H$. rondeletii desova entre el trópico de Cáncer y los $30^{\circ} \mathrm{N}$ entre enero y marzo, en aguas de temperaturas entre los $18^{\circ} \mathrm{y}$ $23^{\circ} \mathrm{C}$. Durante la primavera, la migración comienza hacia aguas más frías encontrándose ejemplares aislados cerca de $\operatorname{los} 38^{\circ} \mathrm{N}$ en aguas de $15,5-18,2^{\circ} \mathrm{C}$; hacia fines de verano puede llegar tan lejos como los $40^{\circ} \mathrm{N}$ (agosto-septiembre). Aunque se desconoce la fecha exacta de captura, sino solo sabe que fue en abril de 1991, sí se conocen los datos de temperatura superficial. Estos datos indican que el pez estuvo nadando en aguas más frías que las mencionadas por otros autores, como rangos conocidos para esta especie. Probablemente $H$. rondeletii visita esporádicamente aguas más frías frente a la costa de Chile, como ha sido el caso del especimen que aquí se presenta.
Hasta hoy, no se conocían especímenes depositados en colecciones chilenas, ya que por error, el ejemplar IZUA-PM-1448 había sido catalogado como Exocoetus volitans Linnaeus, 1758. H rondeletii parece habitar latitudes más ecuatoriales, dadas las referencias hechas por los autores aquí mencionados, a los cuales cabe agregar a Fischer (1978), quien destaca la presencia de $H$. rondeletti en el golfo de México, alrededor de Florida, al norte de Cuba y frente a la costa Atlántica de Estados Unidos, aproximadamente desde $28^{\circ} \mathrm{N}$ hacia el norte. En forma similar, Chirichigno et al. (1982) la mencionan para el Pacífico oriental, incluyendo Hawaii, desde $32^{\circ} 43^{\prime} \mathrm{N}$ hasta $37^{\circ} 00^{\prime} \mathrm{S}$ (zonas de pesca $77 \mathrm{y}$ 87a, b y c de FAO). El registro actual de Ancud, cerca de los $42^{\circ} \mathrm{S}$ parece constituir un nuevo límite sur en su distribución en el océano Pacífico y consecuentemente, el primero en la zona de pesca $87 \mathrm{~d}$ de FAO. Con el presente especimen, existen solamente dos ejemplares de Hirundichthys rondeletii preservados en colecciones chilenas. De las especies nominales de Exocoetidae reconocidas en la ictiofauna chilena (Pequeño, 1989; 1997), solamente ocho están preservadas en tales colecciones; con el agregado de dos ejemplares, a los cuales solamente se les ha podido asignar el género (Tabla 2).

Tabla 2. Antecedentes de peces voladores, familia Exocoetidae, preservados en colecciones chilenas, con datos de colección, número de catálogo, especie (según cada catálogo), localidad de colecta, número de ejemplares $(\mathrm{N})$ y fecha de captura.

Table 2. Data on flying fishes, family Exocoetidae, preserved in Chilean collections, indicating institutional collecion, catalog number, species (according to each catalog), locality, number of specimens (N) and date of capture.

\begin{tabular}{|cccccc|}
\hline Colección & N $^{\text {Catálogo }}$ & Especie & Localidad & N & Fecha \\
\hline MNHNC & P-5816 & Cheilopogon heterurus & Arica & 1 & $1 / 1977$ \\
MNHNC & P-5568 & Cypselurus lineatus & Juan Fernández & 3 & $9 / 3 / 1969$ \\
MNHNC & P-6758 & Cypselurus lineatus & Juan Fernández & 2 & $3 / 1988$ \\
MNHNC & P-5728 & Exocoetus volitans & Juan Fernández & 3 & $9 / 3 / 1969$ \\
MNHNC & P-5844 & Fodiator acutus rostratus & Arica & 1 & $1 / 1977$ \\
MNHNC & P-5853 & Fodiator acutus rostratus & Antofagasta & 1 & $12 / 1976$ \\
IZUC & 4783 & Cypselurus pitcairnensis & Isla de Pascua & $?$ & $3 / 1984$ \\
IZUC & 4451 & Cypselurus lineatus & Frente a Arica & 3 & $20 / 8 / 1972$ \\
IZUC & 4303 & Cypselurus spilonopterus & Isla de Pascua & 1 & - \\
IZUC & H-2893 & Prognichthys sp. & Isla de Pascua & 1 & - \\
IZUA & PM 1448 & Hirundichthys rondeletii & 28 ${ }^{\circ} 55^{\prime}$ S,79º32'W & 1 & $7 / 10 / 1991$ \\
IZUA & PM 2245 & Hirundichthys rondeletii & Ancud & 1 & $4 / 1991$ \\
IZUA & PM 1929 & Cypselurus sp. & Alejandro Selkirk & 1 & $2 / 1995$ \\
UANTOF & IIOA-0932 & Cypselurus heterutus & Norte de Chile & 1 & $15 / 1983$ \\
\hline
\end{tabular}


Kong et al. (1985), no encuentran a $H$. rondeletti, entre la ictiofauna asociada al fenómeno El Niño 1982-1983. Según los datos de la Dirección Meteorológica de Chile (www.shoa.cl), durante el año 1991 no hubo fenómeno de El Niño, iniciándose uno solamente en 1992 y el anterior fue en 1986. Así mismo, los fenómenos de La Niña más cercanos son los de 1988 y 1995. Debido a esto, una ampliación en la distribución geográfica austral de $H$. rondeletii por causa de cambios de temperatura en aguas del Pacífico suroriental es poco probable. En relación con el fenómeno de El Niño, Chirichigno (1998) nombra solo una especie de la familia Exocoetidae, Fodiator acutus (Valenciennes, 1847), que habría ampliado su distribución geográfica en Perú.

Heemstra y Parin (1986), señalan que alcanza alrededor de $25 \mathrm{~cm}$ de LE y es una especie oceánica ampliamente distribuida en aguas subtropicales del norte y del sur de todos los océanos, ausente en los trópicos; común frente al Cabo de Buena Esperanza. Esto último permite concordar nuestro hallazgo de $H$. rondeletti, con otros antecedentes de la especie.

Los resultados obtenidos indican que esta familia de peces ha sido muy poco estudiada en aguas de Chile y, para contribuir a futuros estudios, se ha recopilado la información sobre especímenes preservados en colecciones chilenas (Tabla 2).

\section{AGRADECIMIENTOS}

Se agradece la colaboración del Sr. Jorge Valenzuela, quien donó el espécimen estudiado. A los señores CF Rodrigo Nuñez y Ricardo Rojas (Centro Nacional de Datos Oceanográficos, CENDOC), que facilitaron los registros térmicos del mar. Este estudio se hizo con apoyo del Proyecto S-199911 financiado por la Universidad Austral de Chile al Dr. Germán Pequeño.

\section{REFERENCIAS}

Bahamonde, N. y G. Pequeño. 1975. Peces de Chile, Lista sistemática. Mus. Nac. Hist. Nat., Chile, Publ. Ocas., 21: 1-20.

Bruun, A. 1935. Flying fishes (Exocoetidae) of the Atlantic. Dana Rep., 6:1-106.
Chirichigno, N. 1998. Clave para identificar los peces marinos del Perú. Instituto del Mar del Perú, Callao, 496 pp.

Chirichigno, N, W. Fischer y C.E. Nauen. 1982. INFOPESCA. Catálogo de especies marinas de interés económico actual o potencial para América Latina. Parte II. Pacífico Centro y Suroriental. FAO, Roma, 588 pp.

De Buen, F. 1961. Peces chilenos Beloniformes, Syngnathiformes y Gobiidae. Bol. Soc. Biol., Concepción, 35-36: 81-101.

Dirección Meteorológica de Chile. 2001. En: www.shoa.cl.

Fischer, W. (ed.). 1978. FAO Species identification sheets for fishery purposes western central Atlantic (Fishing Area 31), 2: Carangidae to Hemirhamphidae. Rome.

Fischer, W., F. Krupp, W. Schneider, C. Sommer, K.E. Carpenter y V.H. Niem. 1995. Guía FAO para la identificación de especies para los fines de pesca. Pacífico centro-oriental. Roma, 2: 1091 1095.

Fowler, H.W. 1942. Fishes of Chile, Systematic Catalog. Part 2. Rev. Chil. Hist. Nat., 46-47: 15116.

Heemstra, P.C. y N.V. Parin. 1986. Family No116: Exocoetidae. pp. 391-396. En: M.M. Smith and P.C. Heemstra (eds.). Smith's Sea Fishes. Southern Books Publishers, Johannesburg, 1048 pp.

Kong, I., J. Tomicic y J. Zegers. 1985. Ictiofauna asociada al fenómeno El Niño 1982-83 en la zona norte de Chile. Invest. Pesq., Chile, 32: 215-224.

Leviton, A.E., R.H. Gibbs, E. Heal y C.E. Dawson. 1985. Standards in Herpetology and Ichthyology. Part 1. Standard symbolic codes for institutional resource collections in Herpetology and Ichthyology. Copeia, 3: 802-832.

Mann, G. 1954. La vida de los peces en aguas chilenas. Instituto de Investigaciones Veterinarias y Universidad de Chile, Santiago, 342 pp.

Meléndez, R., O. Gálvez y A. Cornejo. 1993. Catálogo de la colección de peces depositada en el Museo Nacional de Historia Natural de Chile. Mus. Nac. Hist. Nat., Chile, Publ. Ocas., 47: 1-233. 
Parin, N.V. 1960a. The flying fishes (Exocoetidae) of the northwest Pacific. Tr. Inst. Okeanol. Moscow, 31: 205-285.

Parin, N.V. 1960b. Distribution of flyingfishes (Family Exocoetidae) in the western and central parts of the Pacific Ocean. Tr. Inst. Okeanol. Moscow, 41: 153-162.
Parin, N.V. 1969. Ichthyological research on the fourth cruise of the research vessel Akademik Kurchatov in the southeastern part of the Pacific Ocean. Probl. Ichthyol., 9(3): 460-464.

Pequeño, G. 1989. Peces de Chile. Lista sistemática revisada y comentada. Rev. Biol. Mar., Valparaíso, 24(2): 1-132. 